\title{
Adhesive Distribution between Paper Components of Cigarettes*
}

\author{
by \\ B. Köll, S. Starlinger, and B. Eitzinger \\ WFT Research, Fabrikstraße 20, 4050 Traun, Austria
}

\section{SUMMARY}

The objective of this contribution is to characterise the distribution of adhesive between the plug wrap paper and the tipping paper on a finished cigarette. On the one hand, it is well known that this distribution influences various properties of the cigarette, but on the other hand, there are no methods available to completely determine this distribution. The area covered by adhesive, the amount of adhesive, and the thickness and position of the adhesive layer between the plug wrap and the tipping paper were chosen as essential quantities. Image analysis was used to evaluate the area covered by adhesive, and the amount of adhesive between the papers. The thickness and position of the adhesive layer were determined by processing pictures of paper cross-sections obtained with a time-of-flight secondary ion mass spectrometer (TOF-SIMS).

These methods were used to investigate the influence of various parameters on the distribution of adhesive. The parameters chosen are the sizing level of the tipping paper, the viscosity of the adhesive and the temperature of the preheater on the cigarette maker. A statistical analysis of the data reveals some of the complex interactions of these parameters and confirms and quantifies many facts known only from experience in paper-making, paper processing and cigarette manufacturing. [Beitr. Tabakforsch. Int. 20 (2003) 373-380]

\section{ZUSAMMENFASSUNG}

Der Gegenstand dieser Untersuchung ist es, die Verteilung von Leim zwischen dem Filterhüllpapier und dem Mundstückbelagpapier einer Zigarette zu charakterisieren, da zwar bekannt ist, dass diese Verteilung Einfluss auf verschiedenste Eigenschaften der Zigarette hat, es aber mit den bisher zur Verfügung stehenden Methoden nicht möglich war, diese Verteilung messtechnisch zugänglich zu machen. $\mathrm{Zu}$ diesem Zweck werden die von Leim bedeckte
Fläche, die aufgetragene Leimmenge, sowie die Position und Dicke der Leimschicht zwischen Filterhüll- und Mundstückbelagpapier als für die Verteilung charakteristische Größen ausgewählt. Es werden einerseits bildanalytische Verfahren eingesetzt, um die bedeckte Fläche und die Leimmenge am Papier zu erfassen. Andererseits werden die Dicke der Leimschicht und deren Position mit Hilfe eines Flugzeit-Sekundärionen-Massenspektrometers (TOFSIMS) und einer nachfolgenden Verarbeitung der aus der Massenspektrometrie gewonnen Bilder bestimmt.

Die entwickelten Methoden werden zur Untersuchung des Einflusses verschiedener Parameter auf die Leimverteilung verwendet. Als Parameter werden der Leimungsgrad des Mundstückbelagpapiers, die Viskosität des Leims und die Temperatur der Schleifheizung variiert. Eine statistische Analyse der gewonnenen Daten gibt Einblick in das komplexe Zusammenwirken der einzelnen Parameter und zeigt, dass viele aus der Papierherstellung, Papierweiterverarbeitung und Zigarettenherstellung bekannte Erfahrungstatsachen sich mit Hilfe dieser Methoden messtechnisch nachweisen lassen. [Beitr. Tabakforsch. Int. 20 (2003) 373-380]

\section{RESUME}

Dans cette étude la distribution des adhésifs entre le papier à cigarette et le papier manchette d' une cigarette est examinée. D' une part, il est bien connu que cette distribution influence les diverses propriétés d' une cigarette mais d' autre part, les méthodes utilisées jusqu' à présent ne permettent pas de réaliser la mesure de cette distribution. Comme quantités caractéristiques de la distribution sont considérées la surface couverte et la quantité de l' adhésif ainsi que l' épaisseur et la position de la couche d' adhésif entre le papier à cigarettes et les manchettes. La surface couverte et la quantité de l' adhésif entre les papiers ont été évaluées par analyse d' image. L'épaisseur et la position de la couche d' adhésif ont été déterminées par spectroscopie 
Table 1. Paper sizing level, adhesive viscosity and pre-heater temperature combinations chosen for the experiments

\begin{tabular}{llll}
\hline No. & Sizing & Viscosity & Temperature \\
\hline 1 & Unsized & Low & Medium \\
2 & Medium & Low & Medium \\
3 & Full & Low & Medium \\
4 & Unsized & High & Medium \\
5 & Medium & High & Medium \\
6 & Full & High & Medium \\
7 & Medium & Low & Low \\
8 & Medium & Low & High \\
\hline
\end{tabular}

de masse d' ions secondaires à temps de vol (TOF-SIMS) et un traitement consécutif des images obtenues à l' aide de cette méthode.

Ces méthodes ont été utilisées pour évaluer l' influence des divers paramètres sur la distribution de l' adhésif, à savoir le degré de collage de la manchette, la viscosité de l' adhésif et la température du pré-chauffage. Une analyse statistique des résultats obtenus révèle l' interaction complexe de ces paramètres et confirme de nombreux faits connus d' expérience de la fabrication et du traitement ultérieur du papier ainsi que de la fabrication des cigarettes. [Beitr. Tabakforsch. Int. 20 (2003) 373-380]

\section{INTRODUCTION}

One of the most crucial aspects of high-speed cigarette production is the filter attachment process, where the tipping paper is glued to the tobacco rod and the plug wrap paper. A careful balance between paper properties, adhesive properties and machine settings has to be found to minimise the number of defects. One of the most common manufacturing defects is the separation of the tobacco rod and filter after the rolling process. In (1) such defects were investigated by Fourier transform infra-red (FT-IR) microspectroscopy of the adhesive on the tipping paper. Not only do manufacturing defects occur if the properties of the paper and adhesive are not well matched, other parameters of the final cigarette may also be influenced.

It is, for example, well known and has been confirmed by methods of computational fluid dynamics that the distance between the plug wrap and the tipping paper has an influence on the degree of filter ventilation (2). This distance is at least partially determined by the thickness of the adhesive layer.

Therefore it seems desirable, firstly, to have methods available to investigate how the adhesive is distributed between the tipping and the plug wrap paper and, secondly, to know how properties of the paper, properties of the adhesive, and the machine settings influence this distribution. Thus it is the objective of this study to develop methods to determine the adhesive distribution as completely as possible and to investigate how some of the more important parameters influence this distribution. The method chosen in this investigation is a combination of image analysis and an ad- vanced surface analytic technique, known as time-of-flight secondary ion mass spectrometry (TOF-SIMS). While most applications of TOF-SIMS have their origin in the semi-conductor or metallurgical industry, its application has recently spread to many other areas including the paper industry, see, for example, (3) and (4). Its imaging capabilities combined with high mass resolution offer possibilities unachievable by most other methods.

Direct image analysis of tipping paper samples taken from the finished cigarette is used to characterise the area and the amount of adhesive applied to the paper, while surface analysis of paper cross sections with TOF-SIMS is used to determine the position and the thickness of the adhesive layer.

\section{EXPERIMENTAL}

\section{Materials}

To investigate the effect of various parameters on the adhesive distribution between the tipping paper and the plug wrap paper, cigarettes were produced on a standard cigarette maker (Protos 90S) at a speed of 8000 cigarettes per minute. As the main parameters, the sizing level of the tipping paper, the viscosity of the adhesive and the temperature of the pre-heater on the cigarette maker were chosen. These parameters are thought to have the largest influence on the performance of the cigarette maker as far as the filter attachment is concerned. "Sizing" is a process carried out to produce paper with an enhanced resistance to penetration by liquids. This is usually accomplished by adding sizing agents to the paper, such as alkyl ketene dimers (AKD). The resistance to penetration by liquids thereby obtained will be referred to as the "sizing level" and it can, for example, be determined by measuring the $\mathrm{Cobb}_{30}$ values (5) or the contact angle (6). The $\mathrm{Cobb}_{30}$ values of the three tipping papers were $>30 \mathrm{~g} / \mathrm{m}^{2}, 21.7 \mathrm{~g} / \mathrm{m}^{2}$, and $13.4 \mathrm{~g} / \mathrm{m}^{2}$, and the paper grades will therefore be denoted as unsized, medium-sized, and full-sized respectively. Two adhesives with a viscosity of $3000 \mathrm{mPas}$ (Fuller Datac N 2103) and $6700 \mathrm{mPas}$ (Fuller IPACOLL LP 2613/2 PM) respectively, but otherwise similar composition and properties were used in the experiments. These two levels of viscosity will be denoted by low and high viscosity. In order to investigate the adhesive distribution by means of image analysis, the adhesive was coloured in black by adding $0.5 \%(\mathrm{v} / \mathrm{v})$ Fastusol@ Black 18 L (BASF). Care was taken that the colour did not significantly change any of the adhesive properties, except for the colour, and furthermore, of course, that the two adhesives were of the same colour. Finally three different temperatures, $60{ }^{\circ} \mathrm{C}, 125{ }^{\circ} \mathrm{C}$, and $190^{\circ} \mathrm{C}$, denoted as low, medium and high temperature were chosen for the pre-heater.

Three different paper grades, two different adhesives and three temperatures result in a total of 18 possible experiments, of which 8 were chosen as an appropriate subset. All possible combinations of papers and adhesives were tried at medium pre-heater temperature, giving 6 experiments, Nos. 1-6 in Table 1, and two more experiments were added by varying the pre-heater temperature with the mediumsized paper and the low-viscosity adhesive, Nos. 7 and 8 in Table 1. 


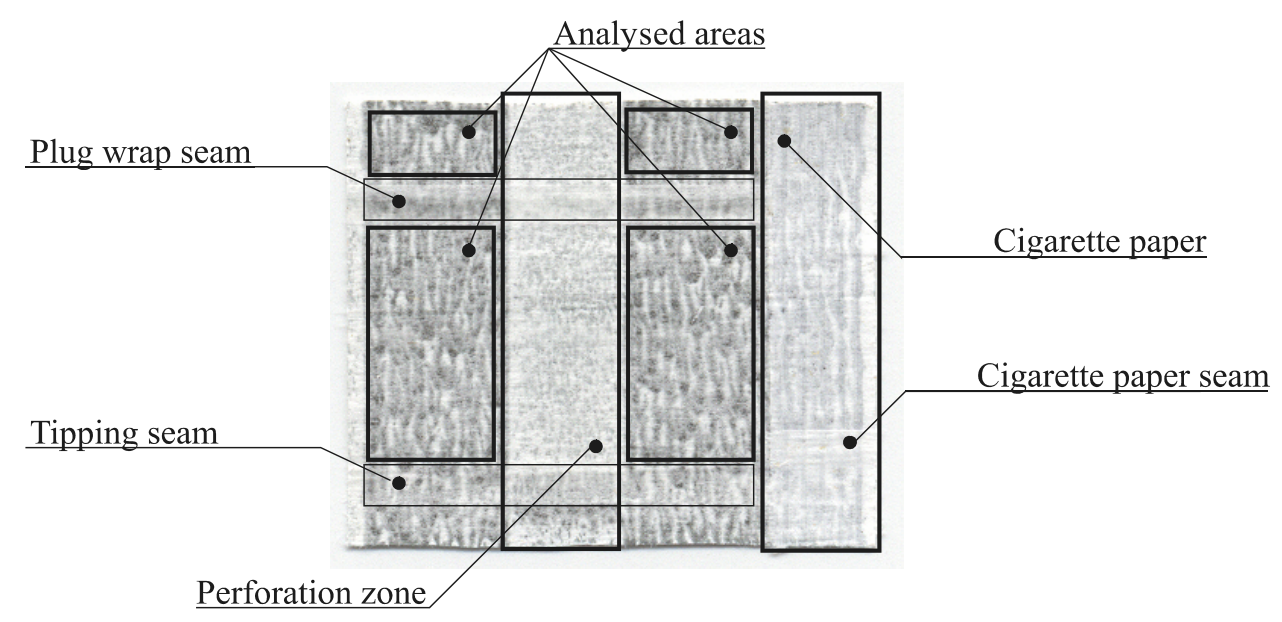

Figure 1. A scanned image of a tipping paper with areas marked for analysis. The perforation zone, adhesive seams and the cigarette paper area were excluded from analysis.
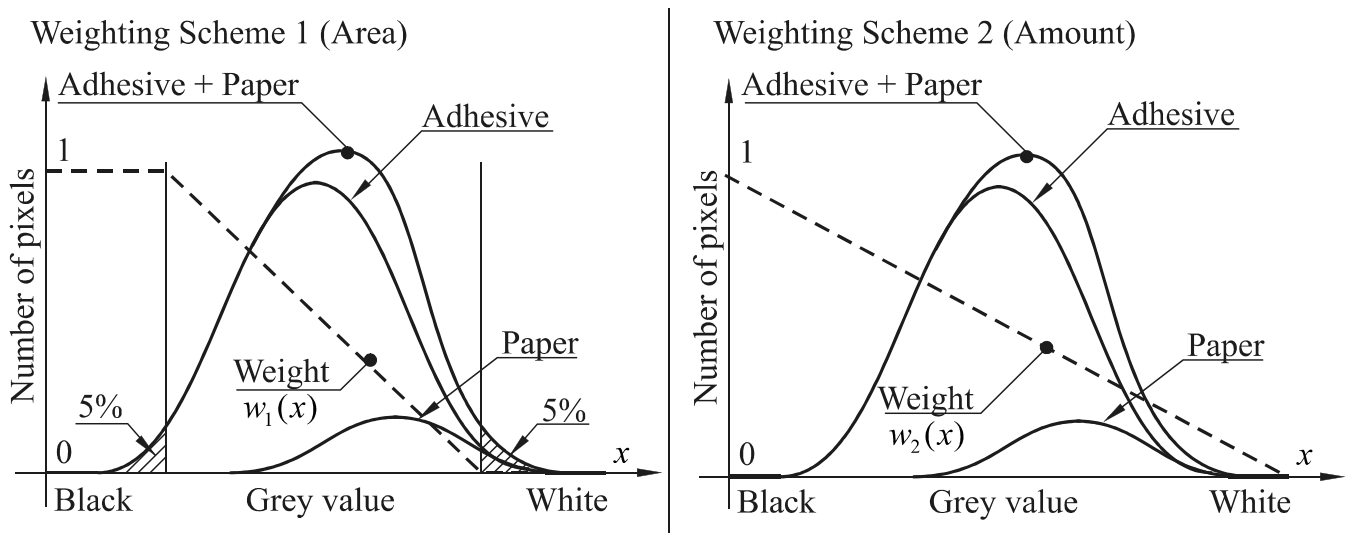

Figure 2. Two weighting schemes were employed in the analysis. Weighting scheme 1, shown on the left, was used to estimate the covered area and weighting scheme 2, shown on the right, was used to estimate the amount of adhesive on the paper.

A sample of cigarettes was taken for each experimental setup after the cigarette maker had reached a steady state.

\section{Methods: Image analysis}

The glued area and the amount of adhesive between the tipping and the plug wrap paper were measured by image analysis. The tipping and the plug wrap paper were carefully removed from the cigarette so that no filter fibres remained on the plug wrap paper. Then a grey-scale $(256$ levels, black $=0$, white $=255$ ) image of the filter side of the tipping paper was acquired at a resolution of $600 \mathrm{dpi}$. Areas not usable for image analysis, i.e. the tipping seam, the perforation zone, and the plug wrap seam, were manually excluded from the analysis. An example of such an image can be seen in Figure 1. From the remaining parts of the image a histogram of grey-scale levels was calculated and further analysed by two different methods.

A typical histogram will appear as shown in Figure 2. The total histogram is the sum of two approximately normally distributed histograms representing the adhesive and the paper. To determine the area covered by adhesive a minimum and a maximum grey-scale level need to be fixed, representing the maximum and minimum amount of adhesive respectively. If we assume that the minimum grey-scale value represents the maximum amount of adhesive, the minimum grey-scale level could be fixed at this value. This procedure, however, is very sensitive to noise in the data, because the minimum value is determined by a single pixel only and may be susceptible to one-pixel artefacts in the image. In order to obtain a minimum level with a lower variance, the level was chosen so that $5 \%$ of the pixels had a smaller grey-scale value than the minimum level. The choice of exactly $5 \%$ is arbitrary, but as long as the percentage is reasonable, for example, between $1 \%$ and $10 \%$, the conclusions remain unchanged. The maximum grey-scale level, representing the minimum amount of adhesive on the paper should be fixed at the maximum of the paper histogram. But as can be seen in Figure 2, this maximum can hardly be determined numerically from the total histogram, especially in the presence of noise. Therefore the maximum level was also fixed by the $5 \%$-rule, that is, $5 \%$ of the pixels had a larger grey-scale value than the chosen maximum level.

To characterise the size of the area of the tipping paper covered by adhesive, the grey-scale levels were weighted according to the following procedure. Grey-scale levels below the minimum level were weighted with 1 , grey-scale levels above the maximum level were weighted with 0 . 

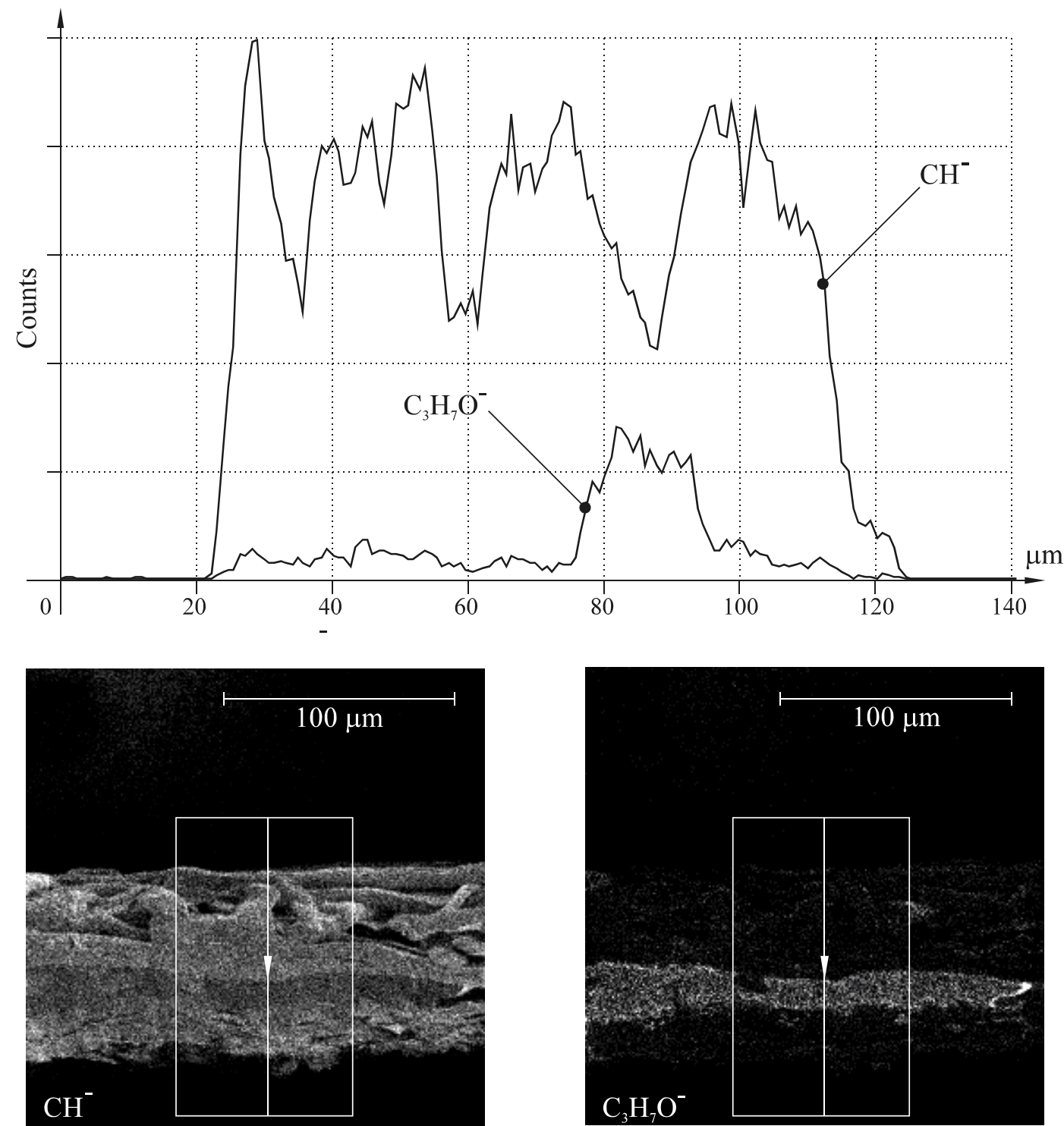

Figure 3. The signal intensities of $\mathrm{CH}^{-}$and $\mathrm{C}_{3} \mathrm{H}_{7} \mathrm{O}^{-}$were detected along a line of $100 \mu \mathrm{m}$ width across the paper caliper. The diagram shows the location of the adhesive layer within the plug wrap/adhesive/tipping system.

$$
w_{1}(x)=\left\{\begin{array}{ccc}
1 & \text { if } & x \leq x_{\min } \\
\frac{x_{\max }-x}{x_{\max }-x_{\min }} & \text { if } & x_{\min }<x \leq x_{\max } \\
0 & \text { if } & x>x_{\max }
\end{array}\right.
$$

Between these two values the weight $w_{1}$ was linearly interpolated, according to the left side in Figure 2 and Equation [1].

The product of the weight and the number of pixels was then summed up over the entire image and divided by the total number of pixels. This ratio determines how much the total histogram is skewed to the right, and hence how much of the paper is still visible. A value of 0.5 corresponds to a perfectly symmetric histogram or as an equivalent to an evenly distributed adhesive layer. As will be observed, real values range between 0.42 and 0.46 , showing that some of the area is not completely covered by adhesive. Furthermore, by this method, the results remain invariant with respect to affine transformations of the grey-scale level and are, therefore, not sensitive to changes of the absolute grey-scale values.
Therefore this ratio is assumed to characterise the size of the covered area, but not the total amount of adhesive on the tipping paper.

To calculate the amount of adhesive on the tipping paper, a different weighting scheme was chosen by simply weighting a grey-scale level of 0 with 1 and a grey-scale level of 255 with 0 and interpolating linearly in between, according to Equation [2] and as shown on the right side in Figure 2.

$$
w_{2}(x)=\frac{255-x}{255}
$$

Again the products of the weight and the number of pixels were totalled and divided by the total number of pixels. This value is sensitive to the absolute grey-value of the image and therefore characterises the total amount of adhesive between the papers.

Both methods were checked by calculating the black area of printed black-and-white paper samples with a known black area. While these simple tests gave accurate values for the black area, we do not claim to actually measure 


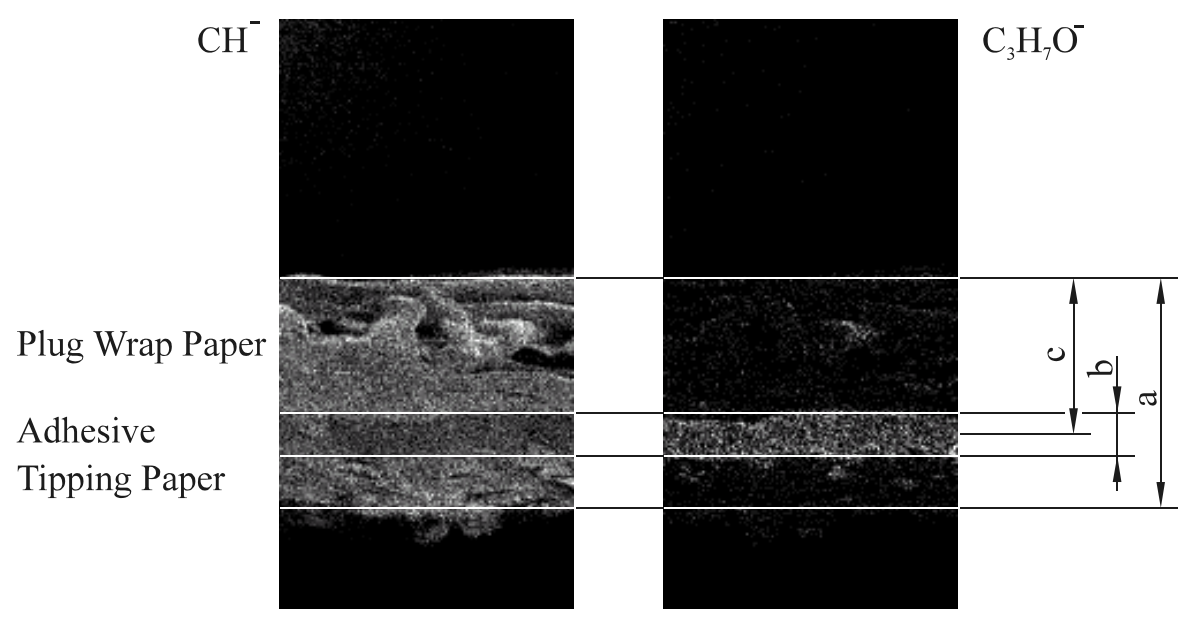

Figure 4. To calculate the position and the width of the adhesive layer, the three values $a, b$ and $c$, were determined from the ion images of $\mathrm{CH}^{-}$and $\mathrm{C}_{3} \mathrm{H}_{7} \mathrm{O}^{-}$. The values $\mathrm{a}, \mathrm{b}$ and $\mathrm{c}$ represent the width of the paper cross-section, the width of the adhesive layer and the distance between the adhesive and the upper edge of the plug-wrap paper respectively.

either the covered area or the amount of adhesive, instead we claim to derive two numbers being strongly related to these quantities, such that we are able to detect qualitative differences. In both cases higher values mean more covered area or a larger amount of adhesive on the paper respectively.

As a second test, the influence of the image resolution was evaluated by analysing several images of tipping papers with resolutions ranging between $75 \mathrm{dpi}$ and $1200 \mathrm{dpi}$. It was found that above 200 dpi the results did not change significantly when the resolution is increased further. Thus a resolution of 600 dpi was chosen.

From each of the eight samples, ten cigarettes were taken randomly from each sample and analysed according to the above procedure. Depending on the relative position of the plug wrap and tipping seam, between two and four areas were usable for image analysis, giving a total of 241 analysed regions, with a combined area of approximately $200 \mathrm{~cm}^{2}$.

\section{Methods: TOF-SIMS}

The position and the thickness of the adhesive layer within the tipping-adhesive- plug wrap system were measured by TOF-SIMS. Therefore, several cigarettes from each experiment were randomly chosen and the papers were carefully separated from the filter plug and manually cut in cross-direction with an uncoated razor blade. Preliminary tests showed that the sample preparation does not affect the distribution of the compounds of interest. In order to keep the cross-section in position during these measurements, the paper samples were mounted in special vice-holders. The resultant surface (cross-section) was analysed using a Physical Electronics TRIFT II TOF-SIMS with an isotopically-enriched ${ }^{115} \mathrm{In}^{+}$liquid metal ion source. An initial analysis was performed at $15 \mathrm{keV}$ in bunched pulse mode in order to identify the peaks at high mass resolution.

To visualise the distribution of the adhesive, the SIMS was then used in the imaging mode, which provides submicrometer lateral resolution.

The data were collected from a $200 \times 200 \mu \mathrm{m}^{2}$ area in the negative SIMS mode using an unbunched $25 \mathrm{keV} / 600 \mathrm{pA}$ primary ion beam. The total primary ion dose was kept below the static limit $\left(10^{12}\right.$ ions $\left./ \mathrm{cm}^{2}\right)$ to avoid ion beam damage in the organic sample. Secondary ions were recorded in the mass range $2.5-1850 \mathrm{~m} / \mathrm{z}$

To overcome charge build-up on the probed insulating paper samples during the SIMS experiments, charge neutralisation was performed between the ion beam pulses by flooding the sample with low energy electrons $(\leq 20 \mathrm{eV})$. Certain fragment ions were selected for the acquisition of ion images to show the distribution of the adhesive and the position of the paper. At least 20 measurements were carried out per sample. A single ion, $m / z, 59\left(\mathrm{C}_{3} \mathrm{H}_{7} \mathrm{O}^{-}\right)$, could be used to visualise the adhesive. Several ion images clearly represented the complete paper cross-section. The fragment ion $m / z, 13\left(\mathrm{CH}^{-}\right)$gave maximum counts per pixel similar to those recorded for the adhesive, which has a favourable effect on the comparability of the images, and subsequently facilitates the evaluation. All ion images were acquired simultaneously and thus showed the distributions of certain compounds for exactly the same area of analysis. Two line scans of $100 \mu \mathrm{m}$ width per acquired section, at the same position for the paper-indicating fragment $\mathrm{CH}^{-}$and the adhesive-characteristic fragment $\mathrm{C}_{3} \mathrm{H}_{7} \mathrm{O}^{-}$, gave the signal intensities along the paper caliper. An example of such line scans for those two fragments can be seen in Figure 3. To detect the upper and lower edge of the paper and to define the position of the adhesive zone, the line scan data were numerically low-pass filtered and the first derivative was calculated, such that the maximum and minimum values of the first derivative clearly indicated paper and adhesive edges. According to Figure 4 and Equation [3], the values for the width $w$ and the position $p$ of the adhesive zone were calculated relative to the measured paper caliper to correct for variations in paper thickness, either caused by natural inhomogeneity or by sample mounting.

$$
p=\frac{c}{a} \quad w=\frac{b}{a}
$$

A total of 328 images was analysed by the above method. 
Table 2. Mean and variance of area, amount, thickness and position. Mean values are dimensionless ratios. The number of measurements is denoted by $N$.

\begin{tabular}{|c|c|c|c|c|c|c|c|c|c|c|c|c|}
\hline \multirow[b]{2}{*}{ No. } & \multicolumn{3}{|c|}{ Area } & \multicolumn{3}{|c|}{ Amount } & \multicolumn{3}{|c|}{ Thickness } & \multicolumn{3}{|c|}{ Position } \\
\hline & $N$ & Mean & Var. $\times 10^{-4}$ & $N$ & Mean & Var. $\times 10^{-4}$ & $N$ & Mean & Var. $\times 10^{-2}$ & $N$ & Mean & Var. $\times 10^{-2}$ \\
\hline 1 & 28 & 0.4501 & 1.198 & 28 & 0.1863 & 4.197 & 44 & 0.1578 & 0.911 & 44 & 0.6509 & 0.924 \\
\hline 2 & 27 & 0.4394 & 1.171 & 27 & 0.1759 & 2.883 & 42 & 0.1773 & 0.510 & 42 & 0.6169 & 0.985 \\
\hline 3 & 35 & 0.4284 & 1.878 & 35 & 0.1802 & 1.646 & 40 & 0.2122 & 0.944 & 40 & 0.6273 & 1.600 \\
\hline 4 & 25 & 0.4345 & 1.031 & 25 & 0.1564 & 1.298 & 40 & 0.2008 & 1.021 & 40 & 0.6822 & 0.460 \\
\hline 5 & 32 & 0.4514 & 1.506 & 32 & 0.1511 & 1.329 & 42 & 0.2265 & 1.361 & 42 & 0.5742 & 0.832 \\
\hline 6 & 36 & 0.4284 & 3.398 & 36 & 0.1707 & 3.124 & 40 & 0.2491 & 1.876 & 40 & 0.5932 & 1.050 \\
\hline 7 & 26 & 0.4461 & 1.075 & 26 & 0.1869 & 2.804 & 40 & 0.2030 & 1.482 & 40 & 0.6163 & 1.601 \\
\hline 8 & 32 & 0.4423 & 0.747 & 32 & 0.1511 & 2.282 & 40 & 0.2020 & 0.641 & 40 & 0.5950 & 0.839 \\
\hline
\end{tabular}

Table 3. Results of the multifactorial analysis of variance (ANOVA) with respect to the influence of sizing level, adhesive viscosity and pre-heater temperature on the glued area, amount of adhesive, and thickness and position of the adhesive layer

\begin{tabular}{llccccc}
\hline Parameters & & Area & Amount & Thickness & Position \\
\hline \multirow{2}{*}{ Sizing } & $p$-value & $\mathbf{1 . 8 9 e - 5}$ & $\mathbf{3 . 9 1 e - 2}$ & $\mathbf{4 . 2 2 e}-\mathbf{3}$ & $\mathbf{3 . 3 8 \mathrm { e } - 2}$ \\
& F-ratio & 11.33 & 3.28 & 5.56 & 3.42 \\
Viscosity & $p$-value & $\mathbf{6 . 8 7 e - 6}$ & $\mathbf{4 . 4 8 \mathrm { e } - \mathbf { 4 }}$ & $\mathbf{4 . 2 9 \mathrm { e } - \mathbf { 2 }}$ & 0.663 \\
& F-ratio & 21.05 & 12.63 & 4.13 & 0.19 \\
\multirow{5}{*}{ Temperature } & $p$-value & $\mathbf{5 . 5 1 e - 1 3}$ & $\mathbf{3 . 2 0 \mathrm { e } - 1 4}$ & 0.373 & 0.089 \\
& F-ratio & 31.42 & 34.97 & 0.99 & 2.44 \\
\hline
\end{tabular}

\section{RESULTS}

The cigarettes were analysed according to the methods described above and the values for covered area, adhesive amount, adhesive thickness and position were obtained as given in Table 2. As described above, all mean values and variances are dimensionless quantities.

A powerful and common method of analysing the data is the analysis of variance (ANOVA). As there are several factors acting on the dependent variables a multifactorial ANOVA was performed. In order for this method to be applicable two preconditions need to be fulfilled, which are in many cases assumed without further investigation. Firstly, the values need to be normally distributed and, secondly, the variance needs to be the same for all samples. While it is reasonable to accept the normality assumption, the second condition of constant variance deserves a closer look. In fact, a statistical test (Bartlett's test) for homogeneity of variance exists and can be applied to this situation (7). This test shows that at a significance level of higher than $99 \%$, the hypothesis of homogeneous variances needs to be rejected. Strictly speaking, for theoretical reasons analysis of variance is not applicable, but practice has shown that inhomogeneous variances do not seriously deteriorate the results. Nevertheless a second, less powerful test, the Kruskal-Wallis test, was also used, because it only needs continuity of the underlying probability distribution as a precondition. It was found, however, that the Kruskal-Wallis test yields the same conclusions, although with slightly different significance levels. Thus only the results for the multifactorial ANOVA are stated in the table. The details of the above mentioned methods can be found in (7).

It was tested whether each of the factors, that is, the papersizing level, the adhesive viscosity, and the pre-heater temperature had a statistically significant influence on the four dependent variables, i.e. area, amount, thickness and position. Table 3 gives the results of the analysis.

\section{DISCUSSION}

The interpretation of Table 3 shows that the experimental conditions do, of course, have an effect on the observed parameters. Among the experimental conditions, the paper-sizing level seems to be the most important, having an influence on all parameters, followed by the adhesive viscosity with a significant influence on adhesive area, adhesive amount, and thickness of the adhesive layer. The pre-heater temperature affects the area and the amount of adhesive on the surface.

\section{Position}

It agrees well with theoretical considerations that the sizing level, but not the viscosity of the adhesive, affects the position of the adhesive layer. This position is determined by an "absorption race" between the tipping paper and the plug wrap paper competing for the available amount of adhesive. As can be seen, the maximum position values, corresponding to more adhesive on the tipping paper side, occur for the unsized tipping papers. In fact, the production of cigarettes with an unsized paper and a low viscosity adhesive proved to be difficult, with frequent breaks of the tipping paper.

The viscosity clearly also affects the speed of absorption but it acts on the absorption speed of both the tipping paper and the plug wrap paper in the same way. This view is also consistent with the fact that sizing level as well as adhesive viscosity significantly influence the thickness of the adhesive layer. The temperature of the pre-heater does not have a significant influence on the position. 


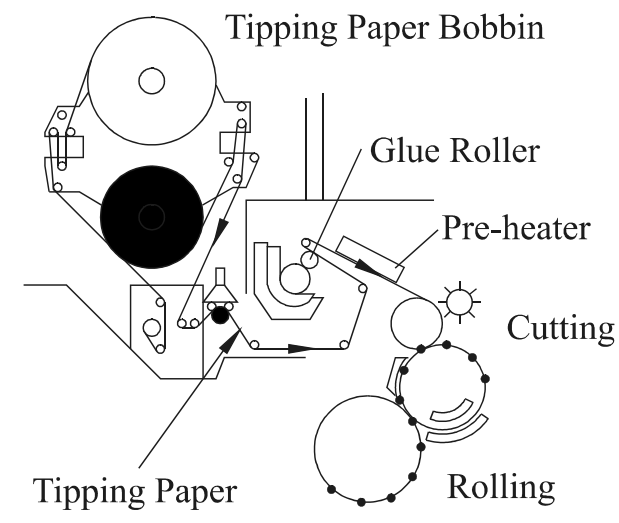

Figure 5. A typical arrangement of the glue roller and the preheater on a cigarette maker

\section{Thickness}

The thickness of the adhesive layer as measured by the above experimental method is determined, on the one hand, by how much adhesive is left in the gap between the plug wrap paper and the tipping paper and, on the other hand, by how much adhesive is being absorbed in the papers and is still visible to TOF-SIMS analysis. The results show that there is a significant increase in thickness when increasing the sizing level of the tipping paper. As full-sized papers do not absorb liquids quickly, a large amount of adhesive remains in the gap between the plug wrap paper and the tipping paper.

Higher viscosity of the adhesive, which is also a significant influence, increases the thickness as well. This result is rather to be expected, for high viscosity usually reduces the speed at which a liquid is absorbed by the paper. It can be imagined that high pre-heater temperatures reduce the adhesive viscosity and therefore also influence the layer thickness, but this could not be confirmed experimentally.

\section{Amount}

As is well known in printing applications, for example in rotogravure printing, viscosity has a large influence on the amount of ink applied to the paper. There is a small range of viscosity values in which the applied amount increases with viscosity, but using inks of too high viscosity significantly reduces the amount transferred to the paper. As far as the present set of data is concerned, there is obviously a large difference in the amount of adhesive on the paper. At the higher viscosity level, significantly less adhesive has been transferred to the paper, which is consistent with the experience in printing applications. One has to be aware, of course, that this result may also be reached if the two adhesives are not of the same colour.

The sizing level does have a significant but quantitatively small influence on the amount of adhesive visible on the surface. It seems that the amount of adhesive transferred to the paper depends largely on the amount on the glue roller and therefore on the viscosity and less on the ability of the paper to absorb liquids, probably because the transfer time of the adhesive is too short for absorption processes to take place.
There is also a significant influence of the pre-heater temperature on the observed amount. From the point of view of technical considerations, this fact seems absurd as the pre-heater is looking in machine direction, mounted after the glue roller, see also Figure 5. The cause is most likely hidden in the image analysis procedure, which gives extremely low variances and is sensitive to changes in colour. Although the influence is statistically significant, it is quantitatively negligible.

Area

From a quantitative point of view, the area covered with adhesive does not change by a technically significant amount. It is mainly owing to the low variance of image analysis measurement procedures that a significant effect could be observed. The maximum change caused by the sizing level is less than 5\% and for the pre-heater temperature it is about $1.5 \%$. Therefore one may conclude that the area covered with adhesive is mainly dependent on the mechanical setup of the glue roller and cannot be influenced by the choice of paper or adhesive.

\section{CONCLUSIONS}

Methods to determine the adhesive distribution between the tipping paper and the plug wrap paper on finished cigarettes were developed. The area covered by the adhesive and the amount of adhesive on the tipping paper were measured by image analysis. The thickness and position of the adhesive layer between the papers were calculated from TOF-SIMS line scans.

The adhesive distribution was investigated for cigarettes produced with different tipping papers, adhesives and machine settings. It was found that the major influences on the adhesive distribution are the sizing level of the tipping paper and the viscosity of the adhesive. Future research could, for example, be aimed at investigating the relationship between the adhesive distribution and the strength of the adhesive bond between the tipping and the plug wrap, thereby directly linking paper and adhesive properties to various manufacturing defects.

Acknowledgements: The authors would like to thank Dr. Hubert Klus of Austria Tabak AG for his support.

\section{REFERENCES}

1. Thompson, M.M. and M.S. Ireland: An application of Fourier transform infra-red (FT-IR) microspectroscopy to the study of cigarette manufacturing defects; Beitr. Tabakforsch. Int. 15 (1992) 59-64

2. Komatsubara O., T. Yoshida, and K. Kaneki: Computational analysis of flow through perforations in tipping paper; Paper presented at the CORESTA Meeting of the Smoke and Technology Groups, Innsbruck, 1999.

3. Conners, T.E. and S. Banerjee (eds.): Surface analysis of paper; CRC Press, New York, N.Y., 1995.

4. Zimmermann, P.A., D.M. Hercules, H. Rulle, J. Zehnpfenning, and A. Benninghoven: Direct analysis of 
coated and contaminated paper using time-of-flight secondary ion mass spectrometry; Tappi Journal 77/8 (1994) 180-186.

5. ISO 535: Paper and board - Determination of water absorptiveness - Cobb method; Reference number ISO 535:1991, International Organization for Standardization, Geneva, Switzerland, 1991.

6. TAPPI T 558 pm-95: Surface wettability and absorbancy of sheeted materials using an automated contact angle tester; Technical Association for the Pulp and Paper Industry, Atlanta, GA, 1995.
7. Hartung, J.: Statistik; 11. Auflage, Oldenbourg Verlag, München, 1998.

Address for correspondence

Bernhard Eitzinger

WFT Research

Fabrikstraße 20

4050 Traun

Austria 